This is a preprint version of the article. It is published in:

International Journal of Selection and Assessment 15 (2007) 2, : 250-262

The final version is available at http://dx.doi.org/10.1111/j.1468-2389.2007.00385.x

The moderating influence of personality and culture on social loafing in typical versus maximal performance situations

\author{
Ute-Christine Klehe \\ Neil Anderson \\ University of Amsterdam \\ University of Amsterdam
}

\author{
Contact: Ute-Christine Klehe \\ Programmagroep Arbeids- en Organisatiepsychologie \\ Universiteit van Amsterdam \\ Roetersstraat 15 \\ 1018 WB Amsterdam \\ The Netherlands \\ Email: U.Klehe@uva.nl
}

Note. This research was funded by a DAAD grant (German Academic Exchange Service) to the first author during her Post-Doc studies at the University of Amsterdam. 


\begin{abstract}
The current paper combines research from personality, cultural, social, and work- and organizational psychology. More precisely, it addresses the motivating effects of situations that either foster or inhibit social loafing under typical versus maximum performance conditions. It further tests how these effects are moderated by the three individual difference variables of conscientiousness, agreeableness, and openness to experience, and the two cultural dimension variables of collectivism and power distance. Results reveal positive main effects for inherently motivating situations, maximum performance conditions, conscientiousness, agreeableness and collectivism, as well as a significant interaction between the degree to which the situation invites social loafing and the typical versus maximum performance condition. These findings thus confirm a possible overlap between the theories of social loafing and of typical versus maximum performance. Finally, power distance showed a number of surprising interactions that may, in part, account for cultural differences found in the social loafing literature. Implications for theory building, empirical research and practice are discussed in the conclusion.
\end{abstract}


The moderating influence of personality and culture on social loafing in typical versus maximal performance situations

The last few years have witnessed an increase in research on performers' reactions to typical versus maximum performance situations (e.g., Kirk \& Brown, 2003; Klehe \& Anderson, 2005; in press; Klehe, Anderson, and Viswesvaran, in press). Typical performance situations represent enduring work situations in which performers are not aware of any performance evaluation or instruction to invest effort, whereas maximum performance situations describe short and evaluative situations during which the instruction to invest effort is quite apparent (Sackett, Zedeck, \& Fogli, 1988). Yet, numerous questions concerning the distinction have not

yet been sufficiently examined, such as the potential overlap with the literature on social loafing (Karau \& Williams, 1993) and the influence of personality and cultural differences on performers' motivation in typical versus maximum performance situations.

Based on an earlier argument (Klehe \& Anderson, 2005), we propose that the effects of typical versus maximum performance conditions interact with incentives towards social loafing as well as with different individual personality and cultural variables. In line with earlier work on typical versus maximum performance (Ployhart, Lim, \& Chan, 2001), which, to our knowledge, has no parallel in the social loafing literature, we also examine the moderating influence of the personality factors conscientiousness, agreeableness, and openness to experience on reported motivation. In integrating these disparate literatures we propose a number of specific hypotheses with regard to main and interaction effects concerning personality and culture upon motivation in typical versus maximum performance situations.

\section{Typical and Maximum Performance}

Performance is generally conceptualized as a function of ability and motivation (Locke, Mento, \& Katcher, 1978; Maier, 1955), the latter being the result of three choices: the choice to 
expend effort (direction), the choice as to which level of effort to expend (level), and the choice to persist in that level of effort (persistence: Campbell, 1990). Yet, the impact of ability and motivation on performance varies. Sackett et al. (1988) introduced the distinction between typical and maximum performance to describe variations in job performance. They argued that during typical performance situations, performers are (a) relatively unaware that their performance may be observed or even evaluated, are (b) not consciously trying to continually perform their 'absolute best', and are (c) working on their task over an extended period of time. In contrast, during maximum performance situations, performers are (a) very well aware of being evaluated, are (b) aware and accept explicit instructions to maximize their effort, and are (c) observed for a short-enough time-period to keep their attention focused on the task at hand. Sackett et al. (1988; see also Sackett, in press) and later DuBois, Sackett, Zedeck and Fogli (1993) proposed that the interplay between both ability and motivation was especially relevant under typical performance conditions. Under maximum performance conditions, however, performance was primarily a function of performers' abilities, since the characteristics of maximum performance situations forced motivation to be high across performers. The choice to perform was constrained to be high due to individuals' knowledge of being monitored. The level of effort was high, since individuals were per definition aware of and accepted the instruction to expend effort. Finally, maximum performance situations should be short enough to ensure that persistence does not become an issue. The basic argument is that during situations of maximum performance, when performers are encouraged to invest their full effort and are evaluated on the basis of their performance, the link between performance and extrinsic rewards becomes highly apparent. This leads performers to be highly motivated, with the resulting performance being a reflection of their ability.

Numerous researchers have stressed the importance of distinguishing between situations of typical and maximum performance (for an overview see Klehe \& Anderson, 2005). Several 
empirical studies using hard as well as soft measures of psychomotor, administrative, and interpersonal performance criteria have provided evidence that typical and maximum performance are related though not interchangeable aspects of performance (Klehe \& Anderson, in press; Klehe \& Latham, 2006; Ployhart, Lim, \& Chan, 2001; Sackett, Zedeck, \& Fogli, 1988). The fact that these data were collected in North-America, East-Asia, and Europe further indicates some cross-cultural generalizability of the typical-maximum performance distinction.

At the same time, it should be noted that typical and maximum performance represent a continuum, making any comparison between the two relative (Sackett et al., 1988). Past research has hardly addressed moderators to typical versus maximum performance, primarily the moderating role of situational factors (i.e., how much the situation invites people to work less under typical performance conditions), personality (e.g., are highly conscientious individuals more likely to resist a possible temptation to invest less effort during typical performance situations; see also Ployhart et al., 2001), or culture (e.g., are the motivating effects of maximum performance conditions more pronounced among individuals scoring high on power distance).

Using an internet-search task within a laboratory setting, Klehe and Anderson (in press) offered a first comprehensive confirmation of Sackett et al.'s (1988) basic assertions. Under maximum performance conditions, participants' motivation increased and the correlation between motivation and performance diminished, while the correlation between ability and performance increased. The same effect should become apparent in a self-assessment of motivation.

Hypothesis 1: People indicate less work-related motivation under typical than under maximum performance conditions.

However, while Klehe and Anderson (in press) showed that typical and maximum performance could feasibly be studied in the laboratory, the generalizability of the results can be 
questioned since performers had little reason to care about the task which was relatively repetitive and of low intrinsic interest to the tested sample. Research on social loafing suggests that results may not always be that clear in case of tasks that hold more intrinsic interest to performers (see also Klehe, Anderson \& Hoefnagels, in press).

\section{Social Loafing}

The first and central characteristic of typical versus maximum performance is the distinction between high (maximum performance) versus low (typical performance) expectations of evaluation (Sackett et al, 1988). Expectation of evaluation is of comparable relevance for the literature on social loafing, which is the tendency to exert less effort on a task performed as an unidentifiable part of a group than when the same task is performed alone (Karau \& Williams, 1993). DuBois et al.'s (1993) argument that "unless one is inviting disciplinary action (in a maximum performance situation), one has little choice but to expend effort on the task in question" (p. 206) is the same argument used for explaining the absence of social loafing when individuals are evaluated. Harkins (1987) and Latané, Williams, and Harkins (1979) proposed that people only engage in social loafing if they think that their performance is not identifiable because they believe that "they can receive neither precise credit nor appropriate blame for their performance" (Latané et al., 1979, p. 830).

Consequently, our knowledge about typical versus maximum performance and its moderators may actually be larger than previously assumed if we are able to adopt findings from the literature on social loafing. In a meta-analysis combining 163 effect-sizes, Karau and Williams (1993) empirically supported the notion that people engage in social loafing if they feel unaccountable for the outcome, but not if they feel that they can be evaluated for their results, thus introducing evaluation potential as a moderator of the social loafing effect. The expectation of being evaluated has been shown to improve performance on simple tasks such as rope pulling 
(Ingham, Levinger, Graves, \& Peckham, 1974), clapping and shouting (Latané et al., 1979), pumping air (Kerr \& Bruun, 1981), and folding paper (Zaccaro, 1979). However, evaluation showed no effect on task performance on more complex tasks such as solving complex mazes (Griffeth, Fichman, \& Moreland, 1989; Jackson \& Williams, 1985). Social loafing is further reduced when individuals (b) work on tasks of high valence (such as high task meaningfulness or personal involvement), (c) work in a group of high valance or when they are given a group-level comparison standard, (d) expect their co-workers to perform poorly, (e) perceive their individual input to be unique, (f) perform in small compared to large groups.

Hypothesis 2: People indicate less work-related motivation in 'unattractive' situations traditionally associated with social loafing (the moderators found by Karau \& Williams, 1993), than in 'attractive' situations.

Given the theoretical link between typical versus maximum performance situations and social loafing outlined above, it is likely that the moderators found for social loafing will equally moderate the impact of typical versus maximum performance situations.

Hypothesis 3: The effects of typical versus maximum performance situation and social loafing will interact with one another. People will only indicate little motivation if they face an unattractive work-situation under typical performance conditions. If either the situation turns into one of maximum performance or becomes more attractive, people will indicate more work-related motivation.

\section{Personality}

Within the social loafing literature, there is a noticeable lack of research on interindividual differences such as personality traits. Research by Ployhart et al. (2001) shows that individuals may react differently to typical versus maximum performance situations based on specific personality characteristics. Additionally, meta-analyses have supported differential links 
between performance and job holders' personality traits (Barrick \& Mount, 1991; Hurtz \& Donovan, 2000; Tett, Jackson, \& Rothstein, 1991), particularly the five-factor model of personality or "Big Five" (Costa, 1996). Although not without criticism (e.g. Block, 1995; Eysenck, 1992; Hough, 1992), the Big Five model has been a relatively well-accepted taxonomy in the field of personality psychology (Goldberg, 1992; John \& Srivastava, 1999; McCrae \& Costa, 1999; Wiggins \& Trapnell, 1997). In the present paper we utilize the Five Factor Model (FFM) as an initial overview structure, although it is likely that combinations of sub-facet dimensions at a finer-grained level of analysis, so-called Criterion-Focussed Occupational Personality Scales (COPS: Ones \& Viswesvaran, 2001; Hough \& Ones, 2001) will prove more parsimonious and powerful in being able to predict outcomes.

Our point here is simply that personality per se has been quite under-researched in typical-maximum performance studies with the work by Ployhart and colleagues (Lim \& Ployhart, 2004; Ployhart et al., 2001) suggesting that the FFM represents perhaps the best initial starting point to begin to tease out overarching effects. Considering the probable impacts of each of the FFM dimensions, we hypothesize the specific relations below in particular for conscientiousness, agreeableness, and openness.

Conscientiousness. Of the five personality traits, conscientiousness has likely received the greatest attention in work and organizational psychology. Barrick et al. (2001) cited 15 metaanalytic studies on personality-performance relationships, the sum of which suggests that conscientiousness consistently predicts work outcomes across jobs. Also labelled conformity (Fiske, 1949), will to achieve (Digman \& Takemoto-Chick, 1981), and prudence (Hogan \& Hogan, 1992), conscientiousness is associated with traits such as dependability and thoroughness, with planning and with being organized, reliable, and responsible (McCrae \& John, 1992), instead of being negligent and careless (Goldberg, 1993). 
The impact of conscientiousness on performance has often been attributed to its link to motivation (Mount \& Barrick, 1995). Highly conscientious performers are more likely to maintain impulse-control or self-discipline, to delay gratifications (Colquitt \& Simmering, 1998) and to persevere longer (Meyer \& Cuomo, 1962) than performers low on conscientiousness. Given this link to motivation, it is reasonable to assume that people scoring high on conscientiousness will be less vulnerable to the demotivating influence of typical performance conditions or of factors promoting social loafing. Consequently, we propose that:

Hypothesis 4a: Conscientiousness will interact with the degree to which a situation invites social loafing (the moderators found by Karau \& Williams, 1993). The higher an individual scores on conscientiousness, the less their reported motivation will depend on the social-loafing potential inherent in the situation.

Hypothesis $4 b$ : Conscientiousness will interact with the typical versus maximum performance condition. The higher an individual scores on conscientiousness, the less their reported motivation will depend on typical versus maximum performance conditions.

Agreeableness. A second personality trait that might be particularly relevant in regard to social loafing is agreeableness, also labeled social adaptability (Fiske, 1949), friendly compliance (Digman \& Takemoto-Chock, 1981), and likeability (Hogan \& Hogan, 1992). Agreeableness refers to such traits as generosity, sympathy, cooperativeness, helpfulness, and courtesy (Digman, 1990). Research by Mount, Barrick, and Stewart (1998) suggests that agreeableness is relevant to job performance in situations needing joint actions and collaboration, as situations characterized by a fairly high level of interpersonal interaction require tolerance, selflessness, and flexibility. This should turn agreeableness into an effective buffer against the demotivating effects of situations that would otherwise invite for social loafing. Consequently, we propose that: 
Hypothesis5: Agreeableness will interact with the degree to which a situation invites social loafing (the moderators found by Karau \& Williams, 1993). The higher an individual scores on agreeableness, the less their reported motivation will depend on the social-loafing potential inherent in the situation.

Openness. Openness to experience, also labeled intellect (e.g. Fiske, 1949; Goldberg, 1992; Saucier \& Goldberg, 2001), culture (Norman, 1963; Tupes \& Christal, 1961), imagination (Goldberg, 1993; Saucier, 1992b), and autonomy (Hendriks, Hofstee, \& Hogan, 1999), encompasses "a broad range of intellectual, creative, and artistic inclinations, preferences, and skills found foremost in highly original and creative individuals" (John \& Srivastava, 1999, p. 114) and "describes the breadth, depth, originality, and complexity of an individual's mental and experiential life" (p. 121).

Openness to experience is distinct from, though related to, general mental ability (Holland, Dollinger, Holland, \& MacDonald, 1995). Thus, it may not surprise that Ployhart et al. (2001) found openness to experience to be related to maximum though not to typical performance. Consequently, we propose that:

Hypothesis 6: Openness will interact with the typical versus maximum performance condition. The higher an individual scores on openness to experience, the more their reported motivation will depend on the typical versus maximum performance situation.

\section{Culture}

Besides personality and the more situational moderators of social loafing, Karau and Williams (1993) also found that the overarching construct of social loafing did not generalize across cultures. While the vast majority of studies on social loafing had been conducted in western cultures, the few studies conducted in eastern cultures indicated that people from an eastern cultural background engaged in less social loafing than people from a western cultural 
background. Thus, social-loafing results indicate international generalizability but with a nested beta-effect (Anderson, 2003) in that correlations between predictors and criteria are in the same direction but differ somewhat in their magnitude across cultures.

The two cultural dimensions most strongly differentiating between eastern and western cultures are individualism and power distance, with eastern countries traditionally scoring considerably lower on the former and higher on the latter dimension than western cultures (Hofstede, online).

Individualism versus collectivism. Individualism versus collectivism refers to the degree to which a culture fosters individualistic tendencies as opposed to group or collectivistic tendencies. Individualistic cultures tend to foster development of autonomous, unique, and separate individuals. In these cultures, the needs, wishes, desires and goals of individuals take precedence over group or collective goals. Collective cultures, in contrast, foster interdependence of individuals within groups. In these cultures, individuals sacrifice their own personal needs and goals for the sake of a common good. There is little theoretical reason to expect individualism versus collectivism to influence reactions to typical versus maximum performance. Yet, Karau and Williams' (1993) findings suggest that individuals with a collectivistic orientation (e.g., Dorfman \& Howell, 1988) would continue to exhibit effort also in situations that would otherwise offer themselves for social loafing:

Hypothesis 7: Individualism / collectivism will interact with the attractiveness of a situation to loaf (the moderators found by Karau \& Williams, 1993). The more individuals express an individualistic cultural orientation, the more their reported motivation will depend on the social-loafing potential inherent in the situation.

Power distance. Power distance refers to the degree to which different cultures encourage or maintain power and status differences between individuals. Cultures high on power distance 
develop rules, mechanisms, and rituals that serve to maintain and strengthen the status relationships among their members. Cultures low on power distance minimize those rules and customs, ignoring, if not eliminating, the status differences between people. In line with the above argument, we propose an interaction between power distance and the attractiveness of the situation to loaf. Since eastern cultures both score higher on power distance and appear less vulnerable to social loafing, such interaction suggests a preventive role of power distance on social loafing.

Hypothesis 8a: Power distance will interact with the attractiveness of a situation to loaf (the moderators found by Karau \& Williams, 1993). The higher an individual scores on power distance, the less their reported motivation will depend on the attractiveness inherent in the situation.

Additionally, power distance is likely to interact with typical versus maximum performance conditions. DuBois et al.'s (1993, p. 206) argument regarding disciplinary actions following maximum performance situations implies a non-negligible power distance between the performer and the person evaluating the performance. Among performers scoring low on power distance, however, the effect of being evaluated during maximum performance situations is likely to be considerably smaller.

Hypothesis $8 b$ : Power distance will interact with the typical versus maximum performance condition. The higher an individual scores on power distance, the more their reported motivation will depend on the typical versus maximum performance situation.

\section{Methods}

\section{Sample}

Participant were 488 undergraduate psychology students of the University of Amsterdam (mean age $=20.9$, S.D. $=4.37$ ) who participated in a series of tests during the traditional "test- 
week", a set of four four-hour testing sessions distributed across four weeks as a means of fulfilling an introductory psychology course requirement. 140 participants were male and 350 female.

\section{Procedure}

Participants answered two questionnaires assessing the personality and cultural variables of interest. Three weeks later, they indicated their motivation on 7-point scales in reaction to a list of 36 hypothetical scenarios under either a typical or a maximum performance condition. The hypothetical scenarios were similar to situational interview questions. The situational interview (Latham, Saari, Pursell, \& Campion, 1980) is a valid predictor of performance (Huffcutt, Conway, Roth, \& Klehe, 2004) under both typical and maximum performance conditions (Klehe \& Latham, , 2006). The underlying mechanism proposed to cause the validity of the situational interview is its ability to assess intentions, a direct antecedent of behaviour (Maurer, Sue-Chan, \& Latham, 1999). Just like situational interview questions, each of the scenarios had been based on a job-analysis conducted in collaboration with five subject matter experts (in this case undergraduate students) to ensure both the understandability and relevance of each item for the targeted (student) population. Items were pre-tested with thirty participants in order to delete items that caused misunderstandings or low interrater agreement.

\section{Experimental conditions}

Participants were randomly assigned to one of four groups in a two-by-two experimental design. One factor, attractiveness of situation to loaf, presented two complementary versions of the same 36-item scenario-list. The other factor represented the typical and maximum performance condition.

Moderators of social loafing. Each scenario list consisted of 36 scenarios with six scenarios each addressing each of the six moderators found in the social loafing literature (task 
Personality, culture, and performance situations 14

valence, group valence and group comparison standard, group size, expected coworker performance, perceived uniqueness of own input, and task complexity; Karau \& Williams, 1993). For each moderator, three scenarios were study-related. The other three scenarios were workrelated, as most Dutch students work part-time beside their studies, primarily in retail and lowerlevel administrative jobs (LSVb, 2004). For each moderator and study/work setting, one scenario focused on the direction of effort: Participants indicated the likelihood with which they would invest effort in the respective situation. One scenario focused on the level of effort (these questions measured how much effort someone would invest in a certain situation) and one scenario focused on the persistence of effort (these questions measured how long someone would invest effort in a certain situation).

Every scenario existed in two versions: One version promoted social loafing (e.g., by indicating that the task was of low task valence) and the other version averted social loafing (e.g., by indicating high task valence). The two complementary versions of the scenario-list always combined eighteen 'social loafing' situations (e.g., situations with low task valence) with eighteen 'no loafing' situations (during which people were expected to loaf less; see Figure 1 for examples). The eighteen scenarios that had been promoting social loafing in Version 1 of the scenario list (dependent variable 1) were adverting loafing in Version 2 of the scenario list. Reversely, the eighteen scenarios that had been adverting social loafing in Version 1 of the scenario list (dependent variable 2) were promoting loafing in Version 2 of the scenario list. We used these two versions of the scenario list and thus the two dependent variables for two primary reasons. First, we wanted to ensure that effects would not be attributable to any possible differences between groups. Second, and more importantly, we did not want to present to one group of participants only scenarios that were inherently motivating in nature and thus preventing social loafing, while another group of participants would only read scenarios that invited for 
social loafing, as such a scenario-list might have appeared unrealistic to participants and might thus created undesirable response effects.

Typical versus maximum performance conditions. Typical versus maximum performance conditions were manipulated via the introduction of the scenario list, following Sackett et al.'s (1988) request for meeting the dimensions of typical versus maximum performance conditions. In the typical performance condition, the scenario list was presented as a survey to learn about students' usual responses to common study- and work-related situations. This instruction did not mention any type of ex- or implicit evaluation and did not request participants to present themselves at their best, but only highlighted the representative nature of the answers required. In contrast, participants in the maximum performance condition were told that the scenario list presented a competitive test of practical intelligence and that they should attempt to score as well as possible on this test, thus making the evaluative nature of the maximum performance condition rather obvious.

Measures

Manipulation check. To test whether the manipulation of the typical versus performance condition had succeeded, participants filled out the second half of the Typical Maximum Performance Scale (TMPS; Klehe \& Anderson, 2004). A successful manipulation was to lead participants of the maximum performance condition to perceive the situation as significantly more evaluative (assessed through four items such as "It was very obvious to me that my performance was being evaluated."), to feel more instructed to invest their full effort (five items such as "I understood and accepted that I should focus my full attention on the task."), and to perceive the maximum performance condition to be more representative of short performance periods (five items such as "This work presents a broad slice of my current activities.", inversely coded), compared to participants of the typical performance condition. Items were scored on a 5- 
Personality, culture, and performance situations 16

point Likert-scale, ranging from 1 (strongly disagree) to 5 (strongly agree). Three $t$-tests revealed that participants in the maximum performance condition scored higher on all three subscales of the TMPS ( $t=5.27$ for evaluation, $t=7.79$ for instruction, $t=2.62$ for duration, all $p<.01$ ), thus indicating that the manipulation of the typical versus maximum performance condition had been successful.

Culture. We measured culture on the individual rather than a collective level. On the individual level, culture is manifested in the cultures that individuals bring with them to f.e. the workplace, based on the cultural milieu in which they were raised and socialized. We used the individualism/collectivism (six items such as "Group welfare is more important than individual rewards.") and power distance subscales (six items such as "Employees should not disagree with management decisions.") of the measure by Dorfman and Howell (1988). Items were scored on a 5-point Likert-scale, ranging from 1 (strongly disagree) to 5 (strongly agree).

Personality. Conscientiousness, agreeableness, and openness to experience were measured with the 5 PFT (Elshout \& Akkerman, 1975), a Dutch measure of the factor model of personality. The 5 PFT assesses each factor and comprises 14 items per scale. Participants rated each item on a seven-point scale regarding how applicable they judged a given description to fit them. Busato, Prins, Elshout, and Hamaker (1999) report that the scales usually demonstrate alpha coefficients above .80 .

\section{Results}

Norms were compared to past American research in order to estimate whether our primarily Dutch sample exhibited any statistically meaningful differences compared to past research conducted with North American samples. No major between-sample differences were found.

Correlations. Means, standard deviations, and correlations for the central study variables 
are presented in Table 1. Cronbach's alphas are shown in the diagonal of Table 1. Reliabilities were generally acceptable or good.

$* * * * * * * * * * * * * * * * * * * * *$

Insert Table 1 about here

$* * * * * * * * * * * * * * * * * * * * *$

Regression analyses. Hierarchical multiple regression analyses on both dependent variables (DV1: reported motivation in the 18 scenarios that promoted social loafing in Version 1 and adverted social loafing in Version 2 of the scenario list; DV2: reported motivation in the 18 scenarios that adverted social loafing in Version 1 and promoted social loafing in Version 2 of the scenario list) revealed that participants' reported motivation was strongly influenced by the performance situations being either one of typical or maximum performance $(\beta=.41$ and .53 , both $p<.01$ ) and whether or not the situation described in the respective scenario invited for social loafing $\left(\beta=-.60\right.$ and $-.46 ; R^{2}=.52$ and .49 for DV1 and DV2, respectively). In both regressions, the interaction term between these two factors $(\beta=.52$ and .29 , both $\mathrm{p}<.05)$ added significant increments in $\mathrm{R}^{2}$. Thus, Hypotheses 1, 2, and 3 were fully supported (see Table 2).

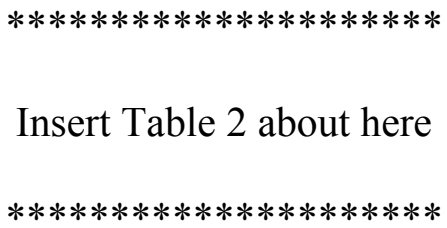

Adding the three personality and two cultural variables into the regression in Step 3 added a significant increment in $\mathrm{R}^{2}$ of .06 and .05 in both regressions $(p<.01)$. Results indicated positive main effects for all three personality variables: conscientiousness $(\beta=.16$ and .13 , both $p<.01)$, agreeableness $(\beta=.07$ and $.08, p<.05)$ and openness to experience $(\beta=.06, p<.10$ and $\beta=.08, p<.05$ ). A positive effect was also found for collectivism (with $\beta=.09$ in both regressions, $p<.05)$. Results for power distance were significantly negative $(\beta=-.08, p<.01)$ in 
the first but nonsignificant $(\beta=-.01, n . s$.$) in the second regression.$

In order to test Hypotheses 4 to 6 , we added the interaction terms between the respective personality constructs and the typical versus maximum performance condition and whether or not the situation invited for social loafing into the regression at Step 4. Results revealed no incremental increase in $\mathrm{R}^{2}$ in either of the two regressions and none of the four interaction terms turned out significant, thus disconfirming Hypotheses 4, 5, and 6.

Finally, we tested for nested beta-effects, that is whether correlations between predictors and criteria differ across cultural variables (Anderson, 2003), by including the interaction terms between cultural factors and performance conditions into the regression analysis in Step 5. We did not include interactions between cultural factors and personality factors, as correlations between those interaction terms and reported motivation were non-significant (see Table 1), indicating that the effects of personality variables on reported motivation remained stable across cultural differences.

Results of both regressions revealed no significant interactions between the attractiveness of the situation to invest effort and individualism/collectivism, thus disconfirming Hypothesis 7. At the same time, results did reveal significant interactions between power distance and the typical versus maximum performance condition in both regression analyses $(\beta=.12$ and .14 , both $p<.05$ ), thus supporting Hypothesis $8 \mathrm{~b}$. In addition, results of the first regression revealed a significant three- way interaction between power distance, the scenarios' attractiveness to loaf and the typical versus maximum performance condition $(\beta=.15, p<.05)$, indicating that the effect for power distance was particularly strong in situations that otherwise presented little attractiveness for high motivation. The second regression showed no such three-way interaction but a significant two-way interaction between power distance and the scenarios' attractiveness to loaf $(\beta=.24, p<.01)$, thus offering partial support for Hypothesis 8 a. Figure 2 shows the 
interacting effect between power distance and attractiveness of the situation to loaf for both Versions of the scenario list. Interestingly, this graph suggests that the interaction between power distance and typical versus maximum performance condition is not due to a high score on power distance, raising participants' motivation in situations that would otherwise invite for social loafing. Instead, individuals scoring high on power distance showed a decrement in motivation in situations that do not invite for social loafing but should be inherently motivating.

$* * * * * * * * * * * * * * * * * * * * *$

Insert Figure 2 about here

$* * * * * * * * * * * * * * * * * * * * *$

We tested the accuracy of this observation via linear regressions of power distance on reported motivation, separated by the version of the questionnaire, that is, whether the respective scenarios invited participants to loaf or not. In both versions of the scenario list, results revealed that power distance had no effect on the degree to which participants indicated that they would invest effort in a non-motivating situation $(\beta=.08$, n.s., and $\beta=.05$, n.s. $)$. Yet, power distance showed a detrimental effect on reported motivation during situations that do not invite for social loafing $(\beta=-.33, p<.01$ and $\beta=-.13, p<.05)$.

\section{Discussion}

The current study examined the interacting effects of typical versus maximum performance conditions, attractiveness of the situation to loaf, personality, and cultural variables on reported motivation on a sample of undergraduate students. Results revealed strong effects for typical versus maximum performance situations, attractiveness to loaf, as well as the interaction between these two factors, indicating that motivation can be raised through either of the two approaches. Counter to our propositions, however, none of the three personality variables of conscientiousness, agreeableness, or openness to experience showed any significant interaction 
with either the degree to which the situations invited social loafing, or with the typical or maximum performance conditions. In contrast, conscientiousness showed a consistent positive impact on reported motivation, a finding reflecting earlier research linking conscientiousness to motivation (Mount \& Barrick, 1995). Also consistent with earlier research, the main-effects of agreeableness and openness to experience on reported motivation were positive, though less stable and not always significant. Of the two cultural variables included, individualism versus collectivism showed a significant main-effect on reported motivation with collectivistic participants indicating higher motivation across experimental conditions. Results, however, did not reveal the proposed interactions with the attractiveness of the situation to loaf (Hypothesis 7). All variance accounted for by interactions with cultural variables was due to participants' different levels of power distance. As proposed, individuals scoring high on power distance reacted more strongly to maximum performance conditions than did individuals who scored low on power distance. Results also confirm the interaction of power distance with attractiveness of the situation to loaf (Hypothesis 8a). However, this effect was not due to individuals scoring high on power distance loafing less, but was due to these individuals reporting less effort even in situations that should be inherently motivating. Thus, our results suggest that it is not individualism versus collectivism that accounts for found cultural differences in social loafing as had been suggested by Karau and Williams (1993), but power-distance. More precisely, a possible explanation for the well reported effect that individuals from eastern cultural background engage in less loafing than individuals from western cultural background may be attributable to a combination of easterners' higher scores on collectivism an on power distance. While a collectivistic orientation in the current study was associated with a net increase in reported motivation across performance conditions, some of this beneficial effect may be reduced particularly in intrinsically motivating situations due to increased scores on power distance. 
The mechanism underlying the conditionally demotivating effects of power distance are open to speculation at this stage and certainly warrant replication and additional research. A possible explanation may be that high power distance is associated with high attention to external sources (such as supervisors) controlling an individual's direction, level, and persistence of effort. According to Deci and Ryan's (Deci, Koestner, \& Ryan, 1999; Ryan \& Deci, 2000) cognitive evaluation thory, a subtheory of their self determination theory, perceptions of external control lower performers' perceived autonomy in their work, leading to a decrease in intrinsic motivation. This way, beneficial motivational effects associated with high collectivism may be reduced particularly in potentially intrinsically motivating situations (e.g., situations of high taskor group valence) due to high power distance. Reversely, another explanation may be that with less power distance, there is more likely to be in-group feeling so that social loafing is reduced ${ }^{\mathrm{i}}$.

On a practical level, results bear a number of implications. First, they confirm the notion that nothing works better in order to motivate employees than to give them motivating working conditions. In some instances, maximum performance conditions may help, yet, these are by definition short in nature and present an entirely extrinsic approach to motivation. An alternative approach are the frequently intrinsically motivating measures identified in the literature on social loafing (Karau \& Williams, 1993), such as attempting to match employees to tasks of their interest and to workgroups of their liking, and to increase their responsibility as well as the visibility of their individual performance.

With a perspective on personnel selection, results suggest the selection not only of highly conscientious individuals, but also of individuals who share a certain degree of collectivist values. Results indicate that more collectivistic oriented individuals are more willing to invest effort and to maintain that effort also in the face of obstacles. In other words, these individuals are more likely to strive for the overall benefits of everyone involved, even though this might 
require personal sacrifices, e.g., in effort and time, but possibly also in other assets, such as personal social standing. At the same time, results suggest some caution in regard to the selection of individuals high on power-distance, even though the reasons underlying this finding are not yet fully known.

\section{Study Limitations and Strengths}

A major limitation of the study is the use of an experimental 'paper people' design. We chose this approach in order to get an overall impression of whether our assumptions were on the right track. While we cannot be certain that our results also apply to actual work situations due to our restricted sample, which consists exclusively of undergraduate university students, a certain amount of generalizability of our findings is implied by the facts that (a) students responded to scenarios that are largely very familiar to them from the study or working lives, and that (b) main effects largely confirmed the findings from earlier research using a wide breadth of different settings (e.g., in regard to the main effects for conscientiousness, agreeableness, and openness).

A cautious note is warranted regarding the lack of support for our hypotheses regarding an interaction between situational and personality variables. When tested with hierarchical moderated multiple regression analysis (Aiken \& West, 1991; Cohen \& Cohen, 1983), the approach most commonly used to test for the presence of interactions, the incremental change in

$\mathrm{R}^{2}$ brought about by adding the cross-product term of the two interacting variables to the equation after each of these variables has already been included individually as a main effect, was not statistically significant. This, however, is not uncommon, as the $F$-test for revealing increments in $\mathrm{R}^{2}$ is sensitive to statistical power. Among the diverse factors contributing to a loss of power are (a) the fact that interactions usually yield very small increments in $\mathrm{R}^{2}$ (Champoux \& Peters, 1987; Chaplin, 1991), especially for personality-variables which themselves usually show only moderate effects on motivation or performance, $(b)$ the fact that the reliabilities of the 
independent variables forming the interaction were far from perfect, and (c) the multicollinearity between the components of the interaction term and the multiplicative composite (Morris, Sherman, \& Mansfield, 1986).

\section{Conclusion}

In overall conclusion, the major results from this study confirm our assumptions regarding a theoretical overlap between typical versus maximum performance and social loafing. They further indicate that performers high on power-distance react more strongly to the distinction between typical and maximum performance than performers low on power-distance. As such, the results bear practical consequences in regard to the selection and motivation of employees, e.g., in selecting for and fostering a sense of conscientiousness and collective orientation and a certain wariness of high power distance as a potentially demotivating factor during otherwise intrinsically motivating work situations.

Additionally, this study adds to the emerging research on the effects of personality in social loafing and in typical versus maximum performance situations (e.g., ForsterLee, in press; Marcus, Goffin, Johnston, \& Rothstein, in press; Ones \& Viswesvaran, in press) by examining potential moderating effects of conscientiousness, agreeableness, and openness to experience. Interactions between personality and situational perceptions of either typical versus maximum performance conditions or the attractiveness of the situation to loaf warrant further studies, perhaps moving toward the inclusion of sub-facets of personality within the FFM structure. The present study highlights the importance of incorporating personality dimensions into both typical-maximum performance and social loafing studies and suggests the need to examine these relations in far greater detail than we have been able to in the single study reported here. Our hope is that future research can begin to tease out these effects and to integrate further the previously disparate fields of personality and culture upon typical-maximum performance situations. 


\section{References}

Aiken, L. S., West, S. G. (1991). Multiple regression: Testing and interpreting interactions. Newsbury Park, CA: Sage Publications.

Anderson, N. (June 2003). The future of selection and assessment: toward a globalized science and practice. Keynote Address to the 5th Australian Industrial and Organizational Psychology Conference, Melbourne, Australia.

Barrick, M. R. \& Mount, M. K. (1991). The Big Five personality dimensions and job performance: A meta-analysis. Personnel Psychology, 44, 1-26.

Block, J. (1995). A contrarian view of the five-factor approach to personality description. Psychological Bulletin, 117, 187-215.

Busato, V. V., Prins, F. J., Elshout, J. J., \& Hamaker, C. (1999). The relation between learning styles, the Big Five personality traits and achievement motivation in higher education. Personality and Individual Differences, 26, 129-140.

Campbell, J. P. (1990). Modeling the performance prediction problem in industrial and organizational psychology. In M. D. Dunnette \& L. M. Hough (Eds.), Handbook of industrial and organizational psychology, Vol. 1 (2nd ed.) (pp. 687-732). Palo Alto, CA: Consulting Psychologists Press.

Champoux, J. \& Peters, W. (1987). Form, effect size, and power in moderated regression analysis. Journal of Occupational Psychology, 60, 243-255.

Chaplin, W. F. (1991). The next generation of moderator research in personality psychology. Journal of Personality, 59, 143-178.

Cohen, J. \& Cohen, P. (1983). Applied multiple regression/correlation analysis for the behaviour sciences. Hilllsdale, NJ: Lawrence Erlbaum Associates. 
Colquitt, J. \& Simmering, M. (1998). Conscientiousness, goal orientation, and motivation to learn during the learning process: A longitudinal study. Journal of Applied Psychology, 83, 654-665.

Costa, P. T. (1996). and personality: Use of the NEO-PI-R in industrial/organisational psychology. Applied-Psychology: An International Review, 45, 225-241.

Deci, E. L., Koestner, R., \& Ryan, R. M. (1999). A meta-analytic review of experiments examining the effects of extrinsic rewards on intrinsic motivation. Psychological Bulletin, 125, $627-668$

Digman, J. (1990). Personality structure: Emergence of the five-factor model. Annual Review of Psychology, 41, 417-440.

Digman, J. M. \& Takemoto-Chock, N. K. (1981). Factors in the natural language of personality: Re-analysis, comparison, and interpretation of six major studies. Multivariate Behavioural Research, 16, 148-170.

Dorfman, P. W. \& Howell, J. P. (1988). Dimensions of national culture and effective leadership patterns: Hofstede revisited. Advances in International Comparative Management, 3, $127-150$.

DuBois, C. L. Z., Sackett, P. R., Zedeck, S., \& Fogli, L. (1993). Further exploration of typical and maximum performance criteria: definitional issues, prediction, and white-black differences. Journal of Applied Psychology, 78, 205-211.

Elshout, J. J. \& Akkerman, A. E. (1975). Vijf persoonlijkheids-factoren Test 5PFT, handleiding[Five personality-factor Test 5PFT, manual]. Nijmegen, Netherlands: Berkhout Nijmegen B.V.

Eysenck, H. J. (1992). Four ways five factors are not basic. Personality and Individual 
Differences, 13, 667-673.

Fiske, D. W. (1949). Consistency in the factorial structures of personality ratings from different sources. Journal of Abnormal and Social Psychology, 44, 329-344.

ForsterLee, R. (in press). Personality, interest, and motivational determinants of maximal and typical performances on a verbal knowledge task. Human Performance.

Gellatly, I. R. and Irving, P. G. (2001). Personality, autonomy and contextual performance of managers. Human Performance, 14, 231-245.

Goldberg, L. R. (1992). The development of markers for the Big Five factor structure. Psychological Assessment, 4, 26-42.

Goldberg, L. R. (1993). The structure of personality traits: Vertical and horizontal aspects. In D. C. Funder, R. D. Parke, C. Tomlinson-Keasey, \& K. Widamin (Eds.), Studying lives through time: Personality and development (pp. 169-188), Washington, D. C.: American Psychological Association.

Griffeth, T. L., Fichman, M., \& Moreland, R. L. (1989). Social loafing and social facilitation: An empirical test of the cognitive-motivational model of performance. Basic and Applied Social Psychology, 10, 253-271.

Harkins, S. G. (1987). Social loafing and social facilitation. Journal of Experimental Social Psychology, 23, 1-18.

Hendriks, A. A. J., Hofstee, W. K. B., \& De Raad, B. (1999). The five-factor personality inventory (FFPI). Personality and Individual Differences, 27, 307-325.

Hofstede, G. (online). http://www.geert-hofstede.com; http://feweb.uvt.nl/center/hofstede/page3.htm ; downloaded Jan. 2007

Hogan, R. T. \& Hogan, J. (1992). Hogan Personality Inventory Manual. Tulsa, OK: 
Hogan Assessment Systems.

Holland, D. C., Dollinger, S. J., Holland, C. J., MacDonald, D. A., (1995). The relationship between psychometric intelligence and the five-factor model of personality in a rehabilitation sample. Journal of Clinical Psychology. 51, 79-88.

Hough, L. M. (1992). The "Big Five" personality variables construct confusion: Description versus prediction. Human Performance, 5, 139-155.

Hough, L.M., \& Ones, D.S. (2001). The structure, measurement, validity, and use of personality variables in industrial, work, and organizational psychology. In N. Anderson, D.S. Ones, H.K. Sinangil, and C. Viswesvaran (Eds.). Handbook of Industrial, Work, and Organizational Psychology (pp. 233-277). London: Sage.

Huffcutt, A. I., Conway, J. M., Roth, P. L., \& Klehe, U.-C. (2004). Evaluation and comparison of the situational and behavior description interview formats. International Journal of Selection and Assessment, 12, 262-273.

Hurtz, G. M. \& Donovan, J. J. (2000). Personality and job performance: The Big Five revisited. Journal of Applied Psychology, 85, 869-879.

Ingham, A. G., Levinger, G., Graves, J., \& Peckham, V. (1974) The Ringelmann effect: Studies of group size and group performance. Journal of Experimental Social Psychology, 10, 371-384.

Jackson, J. M. \& Williams, K. D. (1985). Social loafing on difficult tasks: Working collectively can improve performance. Journal of Personality and Social Psychology, 49, 937942.

John, O. P. \& Srivastava, S. (1999). The "Big Five” trait taxonomy: History, measurement, and theoretical perspectives. In L. Pervin and O. P. John (Eds.), Handbook of 
personality: Theory and research (Second edition; pp. 102-138). New York: Guilford.

Karau, S. J. \& Williams, K. D. (1993). Social loafing: A meta-analytic review and theoretical integration. Journal of Personality and Social Psychology, 65, 681-706.

Kerr, N. L.\& Bruun, S. E. (1981). Ringelmann revisited: Alternative explanations for the social loafing effect. Personality and Social Psychology Bulletin, 7, 224-231.

Kirk, A. K. \& Brown, D. F. (2003). Latent constructs of proximal and distal motivation predicting performance under maximum test conditions. Journal of Applied Psychology, 88, 4049.

Klehe, U.-C. \& Anderson, N. (2004). The Typical-Maximum Performance Scale (TMPS): Assessing perceptions of typical and maximum performance situations. Paper presented at the 19th annual meeting of the Society of Industrial and Organizational Psychology, Chicago, Illinois.

Klehe, U.-C. \& Anderson, N. (2005). The prediction of typical and maximum performance. In A. Evers, N. Anderson, \& O. Smit-Voskuijl (Eds.), Handbook of personnel selection (pp. 331-353). Oxford, UK: Blackwell.

Klehe, U.-C. \& Anderson, N. (in press). Working hard and working smart: motivation and ability during typical and maximum performance. Journal of Applied Psychology.

Klehe, U.-C., Anderson, N., \& Hoefnagels, E. (in press). Social facilitation and inhibition during maximum versus typical performance situations. Human Performance.

Klehe, U.-C., Anderson, N., \& Viswesvaran, C. (Eds.) (in press). Typical versus maximum performance [Special issue]. Human Performance.

Klehe, U.-C. \& Latham, G. P. (2006). What would you do - really or ideally? Constructs underlying the behavior description interview and the situational interview in predicting typical 
versus maximum performance. Human Performance, 19, 357-382.

Latane, B., Williams, K., \& Harkins, S. (1979). Many hands make light the work: The causes and consequences of social loafing. Journal of Personality and Social Psychology, 37, $822-832$.

Latham, G. P., Saari, L. M., Pursell, E. D., \& Campion, M. A. (1980). The situational interview. Journal of Applied Psychology, 65, 422-427.

Lim, B. C. \& Ployhart, R. E. (2004). Transformational leadership: Relations to the FiveFactor model and team performance in typical and maximum contexts. Journal of Applied Psychology, 89, 610-621

Locke, E. A., Mento, A. J., \& Katcher, B. L. (1978). The interaction of ability and motivation in performance: An exploration of the meaning of moderators. Personnel Psychology, $31,269-280$.

LSVb. (2004). Studenten en studentenleven. [students and studentlife]. Utrecht, Netherlands: Onderzoeksbureau van de Landelijke Studenten Vakbond [Researchoffice of the national student organization].

Maier, N. R. F. (1955). Psychology in industry (2nd ed.). Oxford, England: Houghton Mifflin.

Marcus, B. Goffin, R. D., Johnston, N. G. \& Rothstein, M. G. (in press) Personality and cognitive ability as predictors of typical and maximum managerial performance. Human Performance.

Maurer, S. D., Sue-Chan, C., \& Latham, G. P. (1999). The situational interview. In R. W. Eder \& M. M. Harris (Eds.), The employment interview handbook (pp. 159-177). Thousand Oaks, CA: Sage. 
McClelland, G. H. \& Judd, C. M. (1993). Statistical difficulties of detecting interactions and moderator effects. Psychological Bulletin, 114, 376-390.

McCrae, R. R. \& Costa, P. T., Jr. (1999). A fice-factor theory of personality. In L. Pervin \& O. P. John (Eds.), Handbook of personality: Theory and research (Second edition; pp. 139153). New York: Guilford.

McCrae, R. R. \& John, O. P. (1992). An introduction to the five-factor model and its applications. Journal of Personality, 60, 175-216.

Meyer, H. \& Cuomo, S. (1962). Who leaves? A study of background characteristics of engineers associated with turnover. Crotonville, NY: Behavioral Science Research Division, General Electric Company.

Morris, J. H., Sherman, J. D., \& Mansfield, E. R. (1986). Failures to detect moderator effects with ordinary least-squares moderated multiple regression: Some reasons and a remedy. Psychological Bulletin, 99, 282-288.

Mount, M. K. \& Barrick, M. R. (1995). The Big Five personality dimensions: Implications for research and practice in human resources management. In G. R. Ferris (Ed.), Research in personnel and human resources management (Vol. 13, pp. 153-200). Greenwich, CT: JAI Press.

Mount, M. K., Barrick, M. R., \& Stewart, G. L. (1998). Five-Factor Model of personality and performance in jobs involving interpersonal interactions. Human-Performance, 11, 145-165.

Norman, W. T. (1963). Toward an adequate taxonomy of personality attributes: Replicated factor structure in peer nomination personality ratings. Journal of Abnormal and Social Psychology, 66, 574-583.

Ones, D.S. \& Viswesvaran, C. (2001). Personality at work: Criterion-focused 
occupational personality scales (COPS) used in personnel selection. In B.W. Roberts \& R.T. Hogan (Eds.), Applied personality psychology: The intersection of personality and I/O psychology (pp. 63-92). Washington, DC: American Psychological Association.

Ones, D.S. \& Viswesvaran, C. (in press). A research note on the incremental validity of job knowledge and integrity tests for predicting maximal performance. Human Performance.

Ployhart, R. E., Lim, B. C., \& Chan, K. Y. (2001). Exploring relations between typical and maximum performance ratings and the five factor model of personality. Personnel Psychology, 54, 809-843.

Robertson, I. T., Baron, H., Gibbons, P., MacIver, R., \& Nyfield, G. (2000). Conscientiousness and managerial performance. Journal of Occupational and Organizational Psychology, 73, 171-180.

Ryan, R. M. \& Deci, E. L. (2000). Self-Determination Theory and the Facilitation of Intrinsic Motivation, Social Development, and Well-Being. American Psychologist, 55, 68-78.

Sackett, P. R. (in press). Revisiting the Origins of the Typical-Maximum Performance Distinction. Human Performance.

Sackett, P. R., Zedeck, S., \& Fogli, L. (1988). Relations between measures of typical and maximum job performance. Journal of Applied Psychology, 73, 482-486.

Saucier, G. \& Goldberg, L. R. (1991). Lexical studies of indigenous personality factors: Premises, products, and prospects. Journal of Personality, 69, 847-880.

Saucier, G. (1992b). Openness versus intellect: Much ado about nothing? European Journal of Personality, 6, 381-386.

Taylor, P. J., \& Small, B. (2002). Asking applicants what they would do versus what they did do: A meta-analytic comparison of situational and past behaviour employment interview 
questions. Journal of Occupational and Organizational Psychology, 75, 277-294.

Tett, R. P., Jackson, D. N., \& Rothstein, M. (1991). Personality measures as predictors of job-performance: a meta-analytic review. Personnel Psychology, 44, 703-742.

Tupes, E. C. \& Christal, R. E. (1961). Recurrent personality factors based on trait ratings (USAF WADC Tech. Note No. 61-97). Lackland Air Force Base, TX: U. S. Air Force.

Wiggins, J. \& Trapnell, P. D. (1997). Personality structure: The return of the Big Five. In R. Hogan, J. Johnson, \& S. Briggs (Eds.), Handbook of personality psychology (pp. 737-765). San Diego: Academic Press. 
Table 1: Means, Standard deviations, correlations, and internal consistencies of study variables.

\begin{tabular}{|c|c|c|c|c|c|c|c|c|c|c|c|}
\hline & Mean & SD & DV1 & DV2 & Version & $\begin{array}{l}\text { Typ/ } \\
\max \end{array}$ & $\mathrm{C}$ & $\mathrm{A}$ & $\mathrm{O}$ & $\mathrm{IC}$ & $\mathrm{PD}$ \\
\hline DV1 & 4.48 & .77 & $(.85)$ & & & & & & & & \\
\hline DV2 & 4.35 & .70 & $.17 * *$ & $(.80)$ & & & & & & & \\
\hline $\begin{array}{l}\text { Loafing version } \\
\text { (version) }\end{array}$ & .51 & .50 & $.60 * *$ & $-.46^{* *}$ & & & & & & & \\
\hline Typ/max & .50 & .50 & $.41 * *$ & $.53 * *$ & .00 & & & & & & \\
\hline Conscientiousness (C) & .00 & 9.57 & $.24 * *$ & $.19 * *$ & .01 & $.10^{*}$ & (.89) & & & & \\
\hline Agreeableness (A) & .00 & 9.32 & $.19 * *$ & $.17 * *$ & .04 & $.11 *$ & $.27 * *$ & $(.83)$ & & & \\
\hline $\begin{array}{l}\text { Openness to } \\
\text { experience }(\mathrm{O})\end{array}$ & .00 & 9.29 & $.11 *$ & $.12 *$ & .03 & .08 & -.03 & .03 & $(.85)$ & & \\
\hline Individualism (IC) & .00 & .61 & $.15 * *$ & $.21 * *$ & -.03 & $.15 * *$ & $.14 * *$ & $.11 *$ & .05 & $(.71)$ & \\
\hline Power distance (PD) & .00 & .69 & $-.14 * *$ & .01 & -.09 & -.02 & -.04 & $-.18 * *$ & $-.12 * *$ & $.12 * *$ & $(.73)$ \\
\hline
\end{tabular}


Note: $n=488+p<.10 ; * p<.05 ; * * p<.01$

Table 2: Stepwise moderated regressions on both scenario lists.

DV1: List motivating in version $1 \quad$ DV1: List motivating in version 2

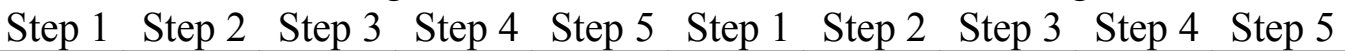

Step 1

version of questionnaire $\quad .60 * * \quad .95 * * \quad .94 * * \quad .95 * * \quad .94 * * \quad-.46 * * \quad-.67 * * \quad-.68 * * \quad-.68 * * \quad-.70 * *$

Typ / max condition $\quad .41^{* *} \quad .77^{* *} \quad .72 * * \quad .74 * * \quad .72 * * \quad .53 * * \quad .32 * * \quad .28 * * \quad .27 * * \quad .26 *$

Step 2

Interaction typ/max \&

version of questionnaire

$$
\begin{array}{llllllll}
-.52 * * & -.51 * * & -.53 * * & -.52 * * & .30 * & .30 * & .30 * & .33 *
\end{array}
$$

Step 3

Conscientiousness (C)

Agreeableness (A)

Openness to exper. (O)

Individualism (IC)

Power distance (PD)

$\begin{array}{lll}.16 * * & .11 * & .13 * \\ .07 * & .05 & .08+ \\ .06+ & .07+ & .06 \\ .09 * * & .09 * * & .09 * \\ -.08 * * & -.08^{* *} & -.03\end{array}$

$\begin{array}{lll}.13 * * & .13 * & .14 * \\ .08^{*} & .11 * & .08+ \\ .09 * * & .06 & .06 \\ .09 * * & .09 * * & .09 * \\ .00 & -.01 & -.23 * *\end{array}$

Step 4

$\mathrm{C} *$ version of questionnaire

$.05 \quad .04$

$.01 \quad .01$

$\mathrm{C} *$ typ/max

A * version of questionnaire

O * typ/max $\begin{array}{ll}.02-.01 & -01\end{array}$

$-.01-.01$
$-.01$

$-.10$

$.24 * *$

$.12 *$

$.14 *$

$-.15 *$
$-.01 \quad-.02$

$.00 \quad .00$

$-.04 \quad-.01$

$.04 \quad .04$

IC * version of questionnaire

\section{3-way PD * typ/max *} version of question.

\begin{tabular}{lllllllllll}
\hline$R$ & $.72 * *$ & $.73^{* *}$ & $.77 * *$ & $.77 * *$ & $.79 * *$ & $.70^{* *}$ & $.71^{*}$ & $.74 * *$ & $.74 * *$ & $.75^{* *}$ \\
Adjusted $R^{2}$ & $.52 * *$ & $.53^{* *}$ & $.59 * *$ & $.59 * *$ & $.61^{* *}$ & $.49 * *$ & $.50^{*}$ & $.54 * *$ & $.54 * *$ & $.55^{* *}$ \\
$\Delta R^{2}$ & $.52 * *$ & $.01 * *$ & $.06 * *$ & .00 & $.03 * *$ & $.50^{* *}$ & $.00^{*}$ & $.05^{* *}$ & .00 & $.02 * *$ \\
\hline
\end{tabular}

Note. $n=488 .+p<.10 .{ }^{*} p<.05 .{ }^{*} * p<.01$. 
Figure 1. Two example scenarios from the scenario list.

(setting: study-related; motivational measure: persistence; moderator: perceived coworker performance - Version 1 labeled the notes as 'really good', Version 2 as 'really poor')

You are sitting in a lecture with a friend. You feel really sick but you want to achieve a good grade in this class. Your friend tells you to go home and says that you can copy his notes. Most of the time his notes are not really good / really poor.

How much longer would you remain in the class?

$\begin{array}{lllllll}1 & 2 & 3 & 4 & 5 & 6 & 7\end{array}$

not at all to the end

(setting: work-related; motivational measure: direction; moderator: group size - Version 1 indicated 'two', Version 2 'twenty' other people)

You are working in an office with two / twenty other people. You were just in a haste to make a few copies when you realize that the copying machine is nearly out of paper.

How likely are you to go and fetch new paper?

$\begin{array}{lllllll}1 & 2 & 3 & 4 & 5 & 6 & 7\end{array}$

not at all likely

very likely 
Figure 2.

Interaction between power distance (taken from one standard deviation below the mean, around the mean, and above the mean) and version of the scenario list.

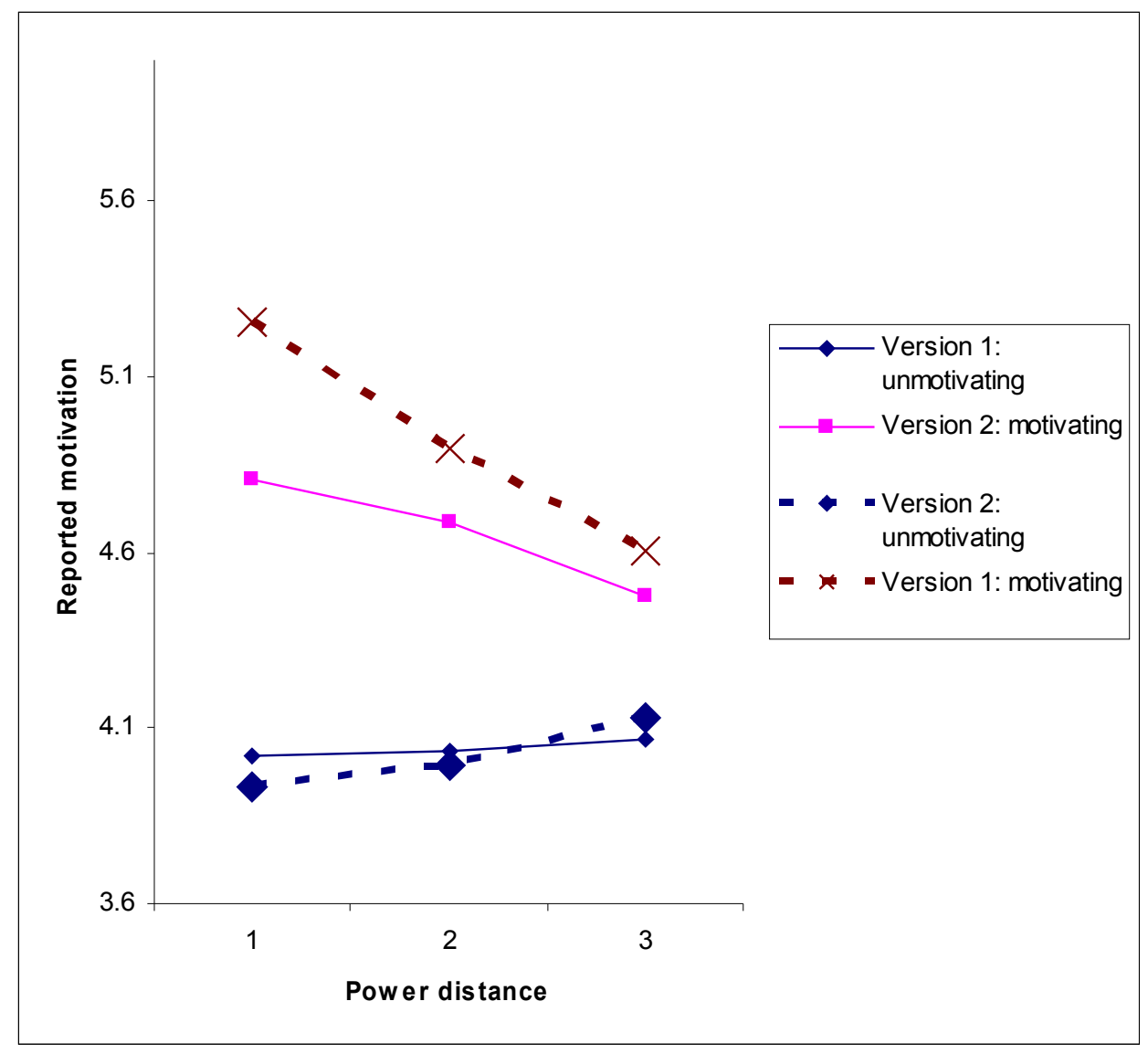


We thank an anonymous reviewer for raising this suggestion. 